\title{
Carbohydrate-binding modules as molecular probes for plant cell wall polymers
}

\author{
Paul Knox, Lesley McCartney \\ Centre for Plant Sciences, University of Leeds, Leeds LS2 9JT, United Kingdom
}

Plant cell walls are complex, polysaccharide-based composites with diverse structural and physiological roles. All plant cells develop a primary cell wall comprised of cellulose microfibrils, hemicelluloses (or cross-linking glycans) and pectic polymers. These walls resist internal hydrostatic forces allowing the development of turgor pressure and are involved in the regulation of cell expansion and cell adhesion. Some cell types, in addition, develop secondary cell walls that are comprised mostly of cellulose and cross-linking glycans and can also be lignified. These walls resist compressive forces, prevent cell collapse and are typical of wood. Several lines of evidence indicate that cell wall polymers are dynamic showing subtle structural changes in response to developmental needs. A measure of the complexity of plant cell walls is that the Arabidopsis genome contains over 700 genes that encode enzymes involved in either the synthesis or metabolism of cell wall polysaccharides [1]. As yet, very few of these genes have been assigned precise biochemical or developmental functions. Structural analyses of cell wall polymers is generally subsequent to homogenization of whole organs resulting in the complete loss of information on the structural variations between cells or within cell walls. One of the best ways to generate information on the growth/development dynamics of polymers or on their role in cell wall architecture is by the use of molecular probes that can bind to specific structural features of polymers. The generation of monoclonal antibodies to a range of cell wall polymers has demonstrated the developmental fine tuning of cell wall polymer structure. For example, antibody probes have revealed extensive modulations of pectic polymer structure during cell development. These include the varied deesterification of homogalacturonan and the extensive developmental regulation of 1,4-galactan and 1,5-arabinan that are structural features of rhamnogalacturonan-I [2]. However, monoclonal antibodies are available for only a small proportion of the polysaccharide structural features that are known to occur in primary and secondary plant cell walls.

Microbial organisms that have the capacity to degrade plant cell walls are widely distributed in nature and have an important role in the turnover of photosynthetically fixed carbon. These organisms secrete batteries of glycoside hydrolase enzymes that target linkages in many cell wall polymers. These enzymes generally have a complex molecular architecture in which non-catalytic domains known as carbohydrate-binding modules or CBMs occur in addition to catalytic domains [3]. It is thought that the main function of CBMs is to facilitate the association of enzymes with substrates enhancing their degradative activity. Currently, there are 42 families of CBMs, assigned by amino acid similarity, in the carbohydrate active enzymes (CAZy: http://afmb.cnrsmrs.fr/CAZY/) database. The first CBMs characterized bound to crystalline cellulose and were initially defined as cellulose-binding domains (CBDs). Recent studies, however, have revealed that CBMs from different families display a wider range of ligand specificity that includes xylans, mannans, glucomannans and $\beta$-1,3-linked glucans in addition to cellulose [3].

The properties of CBM protein modules enables them to be used as molecular probes in a manner equivalent to monoclonal antibodies. We have adapted the use of recombinant CBMs containing histags to a range of assays that are designed to probe plant cell wall polymer structure. Enzyme linked 
detection systems used in nitrocellulose-based assays can provide rapid insight into the binding characteristics and specificities of CBMs and fluorescent tags are used for the localization of CBM ligands in planta [4].

Analysis of sets of cellulose- and xylan-binding CBMs indicate that different CBMs have the capacity to recognize subtle distinctions in these polymers in planta. For example, CBM2b1-2 (from a Cellulomonas fimi xylanase) and CBM6 (from a Clostridium thermocellum xylanase) have similar capacities to bind specifically to a range of xylans as assessed by in vitro assays on nitrocellulose as shown in Fig 1A. However, when applied to sections of a range of plant materials these two CBMs display varying capacities to bind to xylan-containing secondary cell walls as shown in Fig $1 \mathrm{~B}$. CBM2b1-2 binds to all secondary cell walls (where xylans are abundant) in all organs of all angiosperm species examined. In contrast, CBM6 although binding to secondary cell walls of xylem vessels in the pea stem does not bind to the secondary cell walls of the tobacco stem. This may reflect differences in their respective binding sites: CBM2b1-2 has a wide open cleft for binding in contrast to the narrower binding cleft of CBM6 [3]. The lack of CBM6 binding to tobacco stem secondary cell walls is likely to indicate a difference in the disposition of xylan in these walls or distinct taxonomic features that its narrower binding cleft cannot accommodate. Analysis of a range of CBMs in diverse systems should result in an extended set of probes for the analysis of the structures and functions of plant cell wall polymers.

FIG. 1 A: In vitro nitrocellulose-based assay of his-tagged CBM2b1-2 and CBM6 binding to three samples of xylan $(1 \mu \mathrm{g} / \mathrm{dot}$ and 5-fold dilutions). CBMs are detected with an antibodyenzyme detection system. Both CBMs bind equally to all three samples.

B: CBM2b1-2 and CBM6 binding to ligands in sections of tobacco and pea stems. CBM2b1-2 binds to all secondary cell walls of both species. In contrast, CBM6 binds only to the secondary cell walls in pea. It does not bind to xylan of the secondary cell walls in tobacco xylem vessels (single-headed arrow) or phloem fibers (doubleheaded arrow). Scale bar $=100 \mu \mathrm{m}$.

References
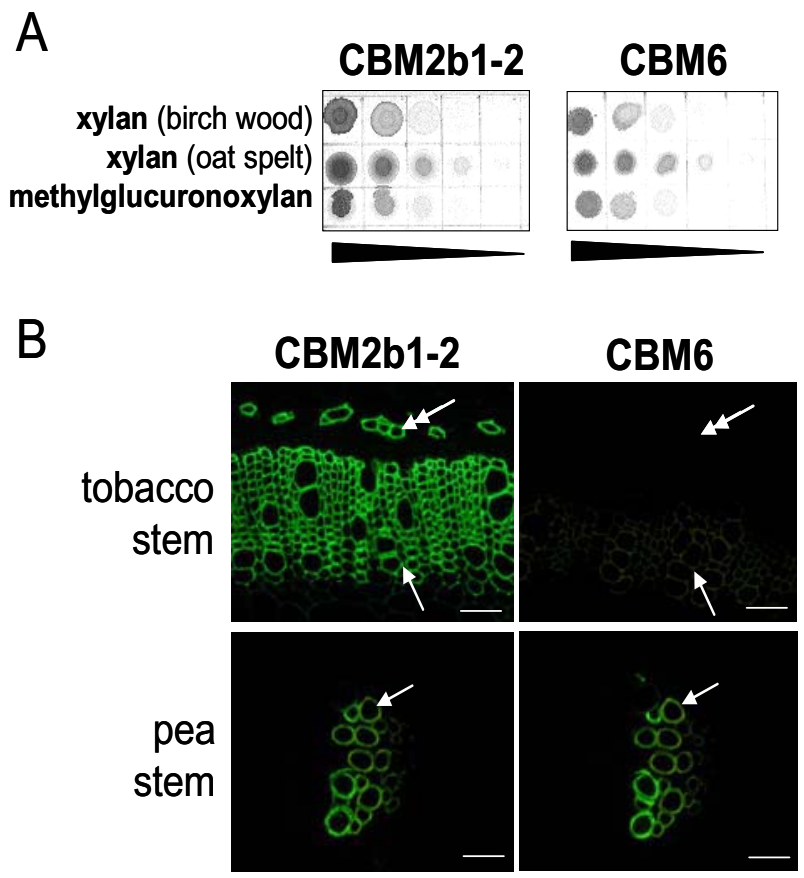

[1] C. Somerville et al. Science 306 (2004) 2206

[2] W.G.T. Willats et al. Plant Mol. Biol. 47 (2001) 9

[3] A.B. Boraston et al. Biochem. J. 382 (2004) 769

[4] L. McCartney et al., Anal. Biochem. 326 (2003) 49

[5] We thank Harry Gilbert, David Bolam and James Flint (University of Newcastle-Upon-Tyne) for CBMs, discussions and insights. This work was supported by the UK Biotechnology \& Biological Sciences Research Council. 